\section{Utilidad de la recolección de orina de dos horas para el diagnóstico del tipo de acidosis tubular renal}

\section{RESUMEN}

La acidosis tubular renal se caracteriza por acidosis metabólica hiperclorémica. El diagnóstico del tipo de acidosis tubular renal se realiza mediante la medición del transporte tubular máximo de bicarbonato y de la capacidad de acidificación urinaria; sin embargo, estas pruebas son invasivas y requieren determinaciones especializadas.

Objetivo: comparar la utilidad de la recolección urinaria de dos horas, una prueba relativamente simple y al alcance de muchos laboratorios, con la medición del transporte tubular máximo de bicarbonato y con la capacidad de acidificación urinaria (procedimientos de referencia) para clasificar el tipo de acidosis tubular renal en pacientes pediátricos.

Material y método: el estudio se realizó en niños con diagnóstico de acidosis tubular renal. El primer día se recolectó la muestra sérica y urinaria de dos horas. Al día siguiente se efectuaron los procedimientos de referencia administrando bicarbonato de sodio en 8 horas; las muestras se colectaron cada hora y se determinaron la reabsorción de bicarbonato y la acidificación urinaria.

Resultados: se incluyeron 19 pacientes y en 17 casos la colección urinaria de dos horas confirmó el diagnóstico de los procedimientos de referencia. La recolección urinaria de dos horas tuvo sensibilidad de 0.94 y especificidad de 0.67 para el diagnóstico de acidosis tubular renal distal.

Conclusión: la recolección de orina de dos horas se realiza en forma menos invasiva y ofrece resultados semejantes a los procedimientos de referencia.

Palabras clave: acidosis tubular renal, pruebas de diagnóstico, niños.
Margarita Irene Rocha-Gómez ${ }^{1}$ Samuel Zaltzman-Girshëvich ${ }^{2}$ Silvestre García-de la Puente ${ }^{3}$

1 Jefe del Servicio de Nefrología Pediátrica y Hemodiálisis, Hospital Regional de Alta Especialidad del Niño Dr. Rodolfo Nieto Padrón.

2 Jefe del Depto. de Nefrología, Instituto Nacional de Pediatría.

${ }^{3}$ Instituto Nacional de Pediatría Nefrología, Metodología de la Investigación.

\section{Utility of two-hour urine collection test for the diagnosis of the type of renal tubular acidosis}

\section{ABSTRACT}

The Renal Tubular Acidosis (RTA) is characterized by hyperchloremic metabolic acidosis with normal anion gap. Diagnosis of RTA type is performed by measuring the maximum bicarbonate tubular transportation (TmHCO3) and urinary acidification capacity (UAC), however these tests are invasive and require specialized determinations.
Recibido: 29 de abril del 2015

Aceptado: 13 de julio del 2015

Correspondencia: Dr. Silvestre García de la Puente Instituto Nacional de Pediatría Insurgentes Sur 3700-C CP 04530, México, D.F.

Tel.: (55) 1084 0900, ext. 1705

garciadelapuente@hotmail.com

Este artículo debe citarse como Rocha-Gómez MI, Zaltzman-Girshëvich S, García-de la Puente S. Utilidad de la recolección de orina de dos horas para el diagnóstico del tipo de acidosis tubular renal. Acta Pediatr Mex 2015;36:322-329. 
Objective: Compare diagnostic utility of a relatively simple and available test (i.e. 2-hour urine collection) with the reference standard of $\mathrm{TmHCO}_{3}$ and $\mathrm{UAC}$ for the classification of RTA.

Material and methods: The study was conducted in children diagnosed with RTA, to evaluate the diagnostic utility of 2-hour urine collection test compared with the measurement of $\mathrm{TmHCO} 3$ and UAC, to establish the type of RTA. The first day, serum and urine sample was collected during a two-hour period. The next day, the reference test was performed by administering sodium bicarbonate in 8 hours. Samples were collected every hour, determining the bicarbonate reabsorption and urinary acidification.

Results: 19 patients were included, in 17 two-hour urinary collection test confirmed the diagnosis made by reference standard tests. Twohour test for the diagnosis of distal RTA had a sensitivity of 0.94 and specificity of 0.67 .

Conclusion: The two-hour urine collection test is performed quickly, less invasive, providing similar results to standard tests.

Key words: Renal tubular acidosis, diagnosis, children.

\section{INTRODUCCIÓN}

La acidosis tubular renal es un síndrome clínico caracterizado por acidosis metabólica hiperclorémica, ésta se produce por una disfunción tubular para la regulación del equilibrio ácidobase con una función glomerular relativamente normal. Esta condición fue designada como acidosis tubular renal en $1951^{1}$ aunque ya existían reportes publicados de acidosis renal. En la literatura especializada esta afección se reporta como poco frecuente; sin embargo, nuestro grupo considera que es relativamente frecuente ya que corresponde a aproximadamente $1 \%$ de los expedientes del Instituto Nacional de Pediatría.

La homeostasis renal ácido-base se divide en dos procesos: la reabsorción del bicarbonato filtrado, que se lleva a cabo fundamentalmente en el túbulo contorneado proximal, y la excreción de ácidos en la nefrona distal..$^{2-4}$ De acuerdo con el tipo de defecto la acidosis tubular renal se clasifica como distal (tipo I) con incapacidad del túbulo distal para la eliminación de iones hidrógeno; como acidosis tubular renal proximal (tipo II) con incapacidad del túbulo proximal para la reabsorción del bicarbonato filtrado; como acidosis tubular renal mixta (tipo III) en donde existe tanto disminución de la secreción distal de hidrogeniones como bicarbonaturia o como acidosis tubular renal hiperpotasémica (tipo IV) con deficiencia o insensibilidad tubular para la acción de la aldosterona ocasionando disminución de la secreción de hidrogeniones y de potasio (acidosis e hiperpotasemia).

En la acidosis tubular proximal el defecto puede ser único; es decir que sólo involucre la reabsorción de bicarbonato, o puede ser parte de una disfunción tubular proximal generalizada (síndrome de Fanconi) en el que hay, además de acidosis metabólica, glucosuria, hiperaminoaci- 
duria y fosfaturia que condicionan hipofosfatemia y raquitismo. ${ }^{5}$ Tanto las acidosis proximales como las distales pueden ser primarias, idiopáticas o secundarias a distintas enfermedades.

El diagnóstico sindromático se efectúa al detectar acidosis metabólica hiperclorémica, es decir con brecha aniónica normal, sin causa sistémica que la explique. El diagnóstico del tipo de acidosis se efectúa con certeza midiendo la reabsorción máxima de bicarbonato $\left(\mathrm{TmHCO}_{3}\right)$ y la capacidad de acidificación urinaria (CAU) cuya utilidad para el diagnóstico y clasificación del tipo de acidosis tubular renal ha quedado demostrada. ${ }^{2}$ Pero se trata de una prueba costosa, de difícil realización y molesta para el paciente. Por otra parte, existen pruebas más sencillas que podrían orientar hacia el tipo de acidosis tubular renal ${ }^{6,7}$ como el $\mathrm{pH}$ urinario, la medición de diversos electrólitos en la sangre y en la orina y la medición de la filtración glomerular.

\section{OBJETIVO}

El objetivo de este estudio fue comparar la utilidad de una prueba relativamente simple y al alcance de muchos laboratorios, que es la recolección urinaria de dos horas, con la reabsorción máxima de bicarbonato y con la capacidad de acidificación urinaria para clasificar el tipo de acidosis tubular renal.

\section{MATERIAL Y MÉTODOS}

Diseño del estudio: observacional, prospectivo, transversal y analítico (de prueba de diagnóstico).

Lugar y población de estudio: el estudio se realizó en la sala de nefrología del Instituto Nacional de Pediatría (México), en el período comprendido entre julio del 2001 y mayo del 2002, en niños con diagnóstico de acidosis tubular renal que se encontraban en tratamiento con soluciones alcalinizantes. El diagnóstico inicial de acidosis tubular renal fue por datos clínicos y acidosis metabólica $\left(\mathrm{pH}\right.$ menor de 7.37, $\mathrm{HCO}_{3}$ menor de $19 \mathrm{mmol} / \mathrm{L}$ y $\mathrm{pCO}_{2}$ menor de $35 \mathrm{mmHg}$ ) con brecha aniónica normal. Se incluyeron pacientes mayores de 2 años, con filtración glomerular normal y firma de consentimiento informado de los padres. Se excluyeron niños con alguna enfermedad activa o con potasio sérico menor de $2.5 \mathrm{mEq} / \mathrm{L}$.

Procedimientos: los procedimientos fueron realizados por un residente de nefrología pediátrica. La muestra fue de casos consecutivos y a los pacientes se les suspendió el tratamiento alcalinizante durante 3 días para condicionar acidosis metabólica; ésta medida no supuso riesgo para los pacientes ya que se trató de un plazo muy corto.

El primer día se efectuó la colección urinaria de los pacientes por aproximadamente 2 horas (marcando los tiempos exactos de la recolección). Se tomó una muestra sanguínea para gasometría venosa, creatinina, sodio $(\mathrm{Na})$, potasio $(\mathrm{K})$, cloro $(\mathrm{Cl})$, calcio $(\mathrm{Ca})$ y fósforo $(\mathrm{P})$. En la orina se determinaron $\mathrm{pH}$, creatinina, $\mathrm{Na}, \mathrm{K}, \mathrm{Cl}$, bicarbonato $\left(\mathrm{HCO}_{3}\right), \mathrm{Ca}, \mathrm{P}$ con los que se obtuvieron los índices para la clasificación del tipo de acidosis tubular renal de acuerdo con el Cuadro 1.

El segundo día se efectuaron los procedimientos de referencia: reabsorción máxima de bicarbonato y capacidad de acidificación urinaria. En el caso de que un niño tuviera bicarbonato $\geq 18 \mathrm{mmol} / \mathrm{L}$ se le administró cloruro de amonio 75 a $150 \mathrm{mEq} / \mathrm{m}^{2}$ a las 03:00 horas del día del estudio mediante sonda nasogástrica. Se canalizó una vena periférica para la administración de soluciones parenterales suficientes para mantener un volumen urinario de $1 \mathrm{a} 5 \mathrm{~mL} / \mathrm{min}$. Solución inicial: glucosada $5 \%, 15 \mathrm{~mL} / \mathrm{kg}$ en 1 hora. Soluciones siguientes: glucosada 5\%, 5 a $7.5 \mathrm{~mL} / \mathrm{kg}$ por hora con $\mathrm{KCl}$ y bicarbonato de sodio. De acuerdo con el bicarbonato basal se 
Cuadro 1. Diagnóstico diferencial de la acidosis tubular renal

\begin{tabular}{|c|c|c|c|c|}
\hline & Proximal & Distal & $\begin{array}{l}\text { Con pér- } \\
\text { dida de } \\
\text { bicarbo- } \\
\text { nato }\end{array}$ & $\begin{array}{l}\text { Hiper- } \\
\text { potasé- } \\
\text { mica }\end{array}$ \\
\hline \multicolumn{5}{|l|}{$\begin{array}{l}\text { Con bicarbona- } \\
\text { to plasmático } \\
\text { bajo }\end{array}$} \\
\hline $\begin{array}{l}\text { Potasio plasmá- } \\
\text { tico }\end{array}$ & $\mathrm{Nl} \circ \downarrow$ & $\mathrm{NI} \circ \downarrow$ & $\mathrm{NI} \circ \downarrow$ & $\uparrow$ \\
\hline Potasio urinario & $\mathrm{NI} \circ \uparrow$ & $\uparrow$ & $\uparrow$ & $\downarrow$ \\
\hline pH urinario & $<5.5$ & $>6$ & $>6$ & $<5.5$ \\
\hline $\begin{array}{l}\text { Brecha anióni- } \\
\text { ca urinaria }\end{array}$ & Negativa & Positiva & Positiva & Positiva \\
\hline Fe de $\mathrm{HCO}_{3}{ }^{*}$ & Variable & $<5 \%$ & $>5 \%$ & $>5 \%$ \\
\hline $\begin{array}{l}\text { Relación Ca/ } \\
\text { creatinina }\end{array}$ & NI & $\uparrow$ & $\uparrow$ & $\mathrm{NI} \circ \uparrow$ \\
\hline
\end{tabular}

calculó el déficit de bicarbonato, para llevarlo a $26 \mathrm{mmol} / \mathrm{L}$ en 6 horas, por medio del siguiente esquema: $\left(26-\mathrm{HCO}_{3}\right.$ real $) \times($ peso $\times 0.5)$; además, se agregó el bicarbonato excretado en el periodo previo. Con relación al potasio sérico, si éste era normal, se administró la pérdida del potasio urinario; si estaba disminuido, se aumentó $20 \%$ de la pérdida y si estaba alto se disminuyó $20 \%$ de la pérdida.

Se canalizó una segunda vía con una aguja tipo mariposa (calibre $24 \mathrm{G}$ ) heparinizada para obtener muestras séricas. Se instaló sonda urinaria tipo Foley para recolección de muestras urinarias en probetas con aceite mineral cubriéndolas con papel parafilm; se mantuvieron en hielo hasta su procesamiento. La orina se colectó cada hora y las muestras sanguíneas a la mitad de las muestras urinarias. Se efectuaron las siguientes determinaciones: en sangre: gasometría, $\mathrm{Na}, \mathrm{K}, \mathrm{Cl}, \mathrm{CO}_{2^{\prime}}$ $\mathrm{Ca}, \mathrm{P}$, creatinina; en orina: $\mathrm{Na}, \mathrm{K}, \mathrm{Cl}, \mathrm{HCO}_{3^{\prime}} \mathrm{Ca}$, $\mathrm{P}$, creatinina, $\mathrm{pH}$, amonio, acidez de titulación.

La duración aproximada de la prueba fue de 8 horas, los pacientes tuvieron dieta normal durante el estudio y una vez concluido egresaron a sus domicilios. Se les prescribió solución alcalinizante en dosis apropiada, por vía oral, dividida en 3 o 4 tomas al día. Durante la prueba se calcularon, cada hora: depuración de creatinina, bicarbonato filtrado, excretado y reabsorbido, acidez de titulación, amonio, brecha aniónica urinaria, fracciones excretadas de sodio ( $\mathrm{FeNa}$ ), de potasio (FeK) y de bicarbonato (FeHCO3), relación calcio/creatinina y porcentaje de reabsorción tubular de fosfatos.

El cálculo de cada una de estas variables se efectuó de las siguientes formas:

\section{a) Depuración de creatinina: \\ $\mathrm{D}_{\mathrm{Cr}}=(\mathrm{U} \times \mathrm{V}) / \mathrm{P} \times 1.73 / \mathrm{SC}$}

Donde:

$U=$ concentración de creatinina en la orina.

$\mathrm{V}=$ volumen urinario/tiempo en minutos.

$\mathrm{P}=$ creatinina plasmática.

$\mathrm{SC}=$ superficie corporal real en $\mathrm{m}^{2}$.

El valor normal es de 80 a $120 \mathrm{~mL} / \mathrm{min} / 1.73 \mathrm{~m}^{2} \mathrm{SC}$ y equivale al filtrado glomerular. ${ }^{8}$

b) Bicarbonato filtrado = depuración de creatinina $\times$ bicarbonato plasmático; bicarbonato excretado $=$ volumen minuto $\times$ bicarbonato urinario; bicarbonato reabsorbido = bicarbonato filtrado - bicarbonato excretado.

Todos los valores son expresados en $\mathrm{mmol} / \mathrm{min}$ por $100 \mathrm{~mL}$ de filtrado glomerular. Cuando se obtiene la máxima cantidad equivale a la reabsorción máxima de bicarbonato. Normal de 2.1 a $2.9 \mathrm{mmol} / \mathrm{min} / 100 \mathrm{~mL}$ de filtrado glomerular. ${ }^{2}$ Un valor inferior indica defecto proximal, ya sea acidosis tubular renal proximal o mixta.

c) Acidez de titulación: se mide titulando la orina con hidróxido de sodio hasta llevar el pH 
urinario a 7.4; se registran los $\mathrm{mEq}$ agregados y se lleva a $\mu \mathrm{Eq} / \mathrm{min} / 1.73$. Normal en acidosis metabólica para niños mayores de 2 años: $\geq 40^{2}$. Si es inferior indica un defecto distal de acidificación en los tres tipos de acidosis tubular renal distal.

d) Amonio: el laboratorio lo determina en $\mu \mathrm{g} /$ $\mathrm{ml}$ y lo expresamos en $\mu \mathrm{Eq} / \mathrm{min} / 1.73$. Normal en acidosis metabólica: $\geq 70^{2}$. Es la principal compensación que hace el organismo ante la acidosis. Está disminuido en caso de acidosis tubular renal distal.

\section{e) Brecha aniónica urinaria $=\left(\mathrm{Na}^{+}+\mathrm{K}^{+}\right)-\mathrm{Cl}$ urinarios.}

Es una medición indirecta del amonio en acidosis metabólica de causa extrarrenal; el cloro es alto, lo que da una brecha aniónica negativa; si es positivo sugiere amonio urinario bajo y acidosis tubular distal. ${ }^{9}$

f) $\mathrm{FeNa}^{+}=\mathrm{U} / \mathrm{P} \mathrm{Na}+\mathrm{U} / \mathrm{P} \mathrm{Cr} \times 100$

Donde:

$U=$ concentración urinaria.

$\mathrm{P}=$ concentración plasmática.

Varía de acuerdo con la dieta o si se administran soluciones endovenosas. En condiciones basales es de aproximadamente 1\%. Es alto en casos de acidosis tubular renal hiperpotasémica por deficiencia de o resistencia a aldosterona. De igual forma se calculan las fracciones excretadas de $\mathrm{K}$ y $\mathrm{HCO}_{3}$.

h) $\mathrm{FeK}^{+}$: normal 10 a $20 \%$; en acidosis tubular hiperpotasémica está disminuída. ${ }^{6}$

i) $\mathrm{FeHCO}_{3}$ : normalmente y en acidosis tubular renal distal es $<5 \%$. En acidosis tubular renal proximal, cuando el bicarbonato plasmático es normal o mayor del umbral plasmático de bicarbonato, es $>15 \%$. En acidosis tubular renal mixta o hiperpotasémica es $>5 \%{ }^{6,7}$

j) Relación Ca/Cr: Ca urinario en mg/dL/creatinina urinaria en $\mathrm{mg} / \mathrm{dL}$. Varía de acuerdo con la edad, en general es menor de 0.2 en escolares y adolescentes y menor de 0.3 en preescolares. ${ }^{10}$ En la acidosis tubular renal está aumentada. ${ }^{6}$

k) $\% \mathbf{R T P}=1-(\mathrm{U} / \mathrm{P}$ fósforo/U/P creatinina $) \times 100$

Normal > 85\%; en casos de acidosis tubular renal con síndrome de Fanconi está disminuída. ${ }^{6}$

Clasificación del tipo de acidosis

Para clasificar el tipo de acidosis tubular renal en función de la recolección urinaria de dos horas se utilizaron los datos enlistados en el Cuadro 1. En la acidosis tubular renal la $\mathrm{FeHCO}_{3}$ habitualmente es mayor de 15\%; sin embargo, cuando el bicarbonato plasmático es menor al umbral plasmático es menor de $5 \%$ ya que se alcanza a reabsorber. Para la clasificación de acuerdo con los procedimientos de referencia se usaron los siguientes criterios:

Acidosis tubular proximal: reabsorción máxima de bicarbonato menor de $2.1 \mathrm{mmol} / 100 \mathrm{~mL}$ de filtrado glomerular; $\mathrm{pH}$ urinario en acidosis metabólica $\leq 5.5$ y capacidad de acidificación urinaria normal.

Acidosis tubular distal: amonio en acidosis metabólica menor de $50 \mathrm{mEq} / \mathrm{min} / 1.73 \mathrm{~m}^{2}$ y pH urinario persistentemente mayor de 6 con potasio sérico normal o bajo.

Acidosis tubular hiperpotasémica: amonio en acidosis metabólica menor de $50 \mathrm{mEq} /$ $\min / 1.73 \mathrm{~m}^{2}$ y $\mathrm{pH}$ urinario en acidosis metabólica $\leq 5.5$ con potasio sérico $\geq 5.5 \mathrm{mEq} / \mathrm{L}$.

Acidosis tubular mixta: reabsorción máxima de bicarbonato menor de $2.1 \mathrm{mmol} / 100 \mathrm{~mL}$ de 
filtrado glomerular, amonio en acidosis metabólica menor de $50 \mu \mathrm{Eq} / \mathrm{min} / 1.73 \mathrm{~m}^{2}$ y $\mathrm{pH}$ urinario persistente mayor de 6 .

El diagnóstico del tipo de acidosis, en ambos casos, fue efectuado en forma ciega por un nefrólogo pediatra.

\section{Análisis estadístico}

La edad se resumió con promedio y desviación estándar; el género con frecuencias y porcentajes. Se calcularon las utilidades diagnósticas para diagnóstico de acidosis tubular renal distal (sensibilidad, especificidad, valores predictivos y razón de probabilidades para pruebas positiva y negativa, con intervalos de confianza de 95\%).

\section{Consideraciones éticas}

El estudio fue aprobado por los comités de investigación y ética del Instituto Nacional de Pediatría. Se explicó el procedimiento a realizar a los padres y firmaron consentimiento informado.

\section{RESULTADOS}

A todos los pacientes que cumplieron con los criterios de selección se les practicaron todos los procedimientos. Se incluyeron 17 mujeres $(89.5 \%)$ y 2 hombres $(10.5 \%)$, con edad de $7.89 \pm 4.01$ años. En el Cuadro 2 se anotan los promedios de las variables del estudio con la desviación estándar entre paréntesis. En la recolección urinaria de dos horas aparecen los valores obtenidos el día previo a la medición de la reabsorción máxima de bicarbonato y de la capacidad de acidificación urinaria y éstos son los valores basales obtenidos con el paciente en acidosis metabólica. Dado que 16/19 pacientes tenían acidosis tubular renal distal el promedio es más representativo de esta condición. El porcentaje de reabsorción tubular
Cuadro 2. Principales variables del estudio

\begin{tabular}{lccc}
\hline Variable & $\begin{array}{c}\text { Recolección } \\
\text { de dos horas }\end{array}$ & $\begin{array}{c}\text { Reabsorción } \\
\text { máxima de } \\
\text { bicarbonato y } \\
\text { capacidad de } \\
\text { acidificación } \\
\text { urinaria }\end{array}$ \\
& & $0.54(0.17)$ \\
Creatinina sérica & $0.50(0.18)$ & $0.3(0.04)$ \\
pH & $7.36(0.03)$ & $7.3(2.5)$ & $15.3(2.2)$ \\
Bicarbonato sérico & $19(2.5)$ & $1.01(1.2)$ \\
Fe bicarbonato & $0.42(0.60)$ & $6.2(0.7)$ \\
pH urinario & $6.5(0.7)$ & $25.1(21.4)$ \\
Acidez titulable & $22.4(31.5)$ & $42.1(37.1)$ \\
Amonio & $24.5(22)$ & $15.6(23)$ \\
Brecha aniónica urinaria & $42.5(28.4)$ & $3.6(0.8)$ \\
Potasio sérico & $3.9(0.6)$ & $(17.3)$ & $32.1(52.4)$ \\
Fe potasio & $16.1(0.9)$ & $0.45(0.5)$ \\
Relación calcio/creatinina & $0.24(0.1)$ & $85.5(8.2)$ \\
\% RTP & $90.4(10.1)$ &
\end{tabular}

Fe: fracción excretada; \% RTP: porcentaje de reabsorción tubular de fosfatos.

de fosfatos muestra una desviación estándar amplia por tratarse de un paciente con acidosis tubular renal proximal y síndrome de Fanconi. La reabsorción máxima de bicarbonato fue de 2 en acidosis tubular renal proximal, de 1.9 en acidosis tubular renal mixta y de 2.3 en acidosis tubular renal distal.

En la prueba de reabsorción máxima de bicarbonato y capacidad de acidificación urinaria 16 pacientes obtuvieron diagnóstico de acidosis tubular renal distal, 2 de acidosis tubular renal mixta y 1 de acidosis tubular renal proximal. En la prueba de dos horas sólo hubo divergencia en dos casos: uno se diagnosticó como acidosis tubular renal proximal cuando en realidad era distal y el segundo se diagnosticó como acidosis tubular renal distal pero ésta era mixta.

Durante la realización de las pruebas no se reportaron complicaciones ni hubo la necesidad de suspender el estudio en ningún paciente por alguna manifestación metabólica que comprometiera su salud. 
Para el diagnóstico de acidosis tubular renal distal, que es la forma más frecuente, en el Cuadro 3 se muestran sensibilidad, especificidad, razón de probabilidades para pruebas positiva y negativa (con intervalos de confianza de 95\%) y valores predictivos.

Cuadro 3. Utilidad de los procedimientos para el diagnóstico de la acidosis tubular distal

\begin{tabular}{lccc}
\hline \multicolumn{4}{c}{$\begin{array}{c}\text { Reabsorción máxima de } \\
\text { bicarbonato y capacidad de } \\
\text { acidificación urinaria }\end{array}$} \\
Prueba 2 horas & Sí & No & Total \\
Sí & 15 & 1 & 16 \\
No & 1 & 2 & 3 \\
Total & 16 & 3 & 19
\end{tabular}

Sensibilidad: 0.94 (IC 95\% 0.82, 1)

Especificidad: 0.67 (IC 95\% 0.14, 1)

Valor predictivo positivo: 0.94

Valor predictivo negativo: 0.67

Razón de probabilidades para un resultado positivo 2.85 (IC 95\% 0.57, 14.24)

Razón de probabilidades para un resultado negativo 0.09 (IC 95\% 0.008, 0.47)

\section{DISCUSIÓN}

Fisiopatológicamente, las acidosis tubulares renales son producidas o por un defecto en el túbulo proximal para reabsorber el bicarbonato filtrado o por un defecto en el túbulo distal para secretar ácido. Las mediciones de la reabsorción máxima de bicarbonato y de la capacidad de acidificación urinaria son las pruebas de referencia para su diagnóstico. No obstante, implican una gran cantidad de equipo y personal para su realización, así como el malestar generado al paciente por los procedimientos y por el tiempo requerido para su realización.

Buscamos un método que reduzca todos los inconvenientes de los procedimientos mencionados sin disminuir la capacidad de diagnóstico; por ello se propuso la recolección de orina de dos horas para diagnosticar acidosis tubulares renales en pacientes ambulatorios del servicio de nefrología.

Al comparar los diagnósticos obtenidos en cada prueba se encontró un error general de 10.5\% en aquellos obtenidos mediante la recolección de orina de dos horas, lo cual permite establecer que se trata de una prueba útil, con un margen de error que justifica su uso pues permite, en la gran mayoría de los casos, establecer el diagnóstico y el tipo de acidosis tubular renal en forma rápida y con menores invasión y molestia a los pacientes.

Se han recomendado algoritmos para el diagnóstico del tipo de acidosis tubular renal $\left.\right|^{6,7,11,12}$ que incluyen, en primera instancia, tomar simultáneamente muestras sanguíneas semejantes a las tomadas en nuestro estudio y determinar en una sola muestra de orina fresca $\mathrm{pH}$ y electrólitos urinarios. Estos resultados posteriormente son complementados con colección urinaria de 24 horas para determinaciones de excreciones de calcio, creatinina y citratos, con lo que generalmente se hace el diagnóstico de la acidosis tubular renal y su tipo. En casos especiales se pueden efectuar otras pruebas, algunas de ellas invasivas, como la determinación de la $\mathrm{pCO}_{2}$ urinaria después de una carga con bicarbonato (una diferencia de $\mathrm{pCO}_{2}$ urinaria- $\mathrm{pCO}_{2}$ sanguínea menor de $20 \mathrm{mmHg}$ confirma la acidosis tubular renal distal) o la medición del pH urinario mínimo, ya sea después de la administración de furosemida con un mineralocorticoide o con la inducción de acidosis metabólica con cloruro de amonio (un pH urinario mayor de 6 confirma la acidosis tubular renal distal).

La ventaja de la recolección urinaria de dos horas es que las únicas molestias para el paciente son la toma de sangre y la recolección corta de orina que permiten medir la filtración glomerular. No utilizamos las determinaciones de citratos 
pues, si bien en la acidosis tubular renal distal hay hipocitraturia, una determinación aislada puede dar resultados no del todo confiables; tampoco utilizamos las mediciones de $\mathrm{pCO}_{2}$ urinaria después de la carga de bicarbonato ya que pensamos que las mediciones de la reabsorción máxima de bicarbonato y de la capacidad de acidificación urinaria son suficientes para diagnosticar con certeza el tipo de acidosis tubular renal.

Pensamos que cuando existan datos clínicos para sospechar acidosis tubular renal la primera etapa consiste en efectuar gasometría y electrólitos séricos para establecer el diagnóstico de acidosis metabólica con brecha aniónica normal. Si es el caso efectuar la colección urinaria de dos horas con determinaciones simultáneas en sangre y suero señaladas en la metodología; con ello podríamos clasificar adecuadamente casi $90 \%$ de los casos.

Las limitaciones de este estudio son lo pequeño de la muestra (que se traduce en intervalos de confianza amplios) y que no se incluyeran más pacientes con acidosis tubulares renales mixta e hiperpotasémica. El género no es representativo en este estudio ya que se seleccionaron más niñas debido a la facilidad para instalar la sonda vesical.

\section{REFERENCIAS}

1. Pines $\mathrm{KL}$, Mudge $\mathrm{GH}$. Renal tubular acidosis with osteomalacia. Am J Med 1951;11:302-11.

2. Edelmann CM JR, Rodríguez Soriano J, Boichis $H$, Gruskin $A B$, Acosta MI. Bicarbonate reabsorption and hydrogen ion excretion in normal infants. J Clin Inv 1967;46:1309-17.

3. Du Bose JR. Reclamation of filtered bicarbonate. Kidney Int 1990;38:584-89.

4. Wrong O. Distal renal tubular acidosis: the value of urinary $\mathrm{pH}, \mathrm{pCo} 2$ and $\mathrm{NH} 4$ measurements. Pediatr Nephrol 1991;5:249-55.

5. Petra S. Nadri G, Clowdhary H, Pemde HK, Singh V, Chandra J. Idiopathic Fanconi'syndrome with nephrogenic diabetes insipidus in a child who presented as vitamin D resistant rickets-a case report and review of literature. J Pediatr Endocrinol Metab 2011;24:755-57.

6. Bagga A, Sinha A. Evaluation of renal tubular acidosis. Indian J Pediatr 2007;74:679-86.

7. García de la Puente S. Acidosis tubular renal. Acta Pediatr Mex 2006;27:268-78.

8. Stevens LA, Levey AS. Measure GFR as a confirmatory test to estimating GFR. J Am Soc Nephrol 2009;20:2305-13.

9. Halperin ML, Richardson RM, Bear RA, Magner PO, Kamel $\mathrm{K}$, Ethier J. Urine ammonium: the key to the diagnosis of distal renal tubular acidosis. Nephron 1988;50:1-4.

10. Sáez-Torres C, Rodrigo D, Grases F, García-Raja AM, Gómez C, \& Lumbreras J, Frontera G. Urinary excretion of calcium, magnesium, phosphate, citrate, oxalate, and uric acid by healthy schoolchildren using a 12-h collection protocol. Pediatr Nephrol 2014;29:1201-08.

11. Chan JCM, Santos F. Renal tubular acidosis in childhood. World J Pediatr 2007;3:92-97.

12. Gil-Peña H, Mejía N, Santos F. Renal tubular acidosis. J Pediatr 2014;164:691-8. 